\title{
Quartet of tau-leptons and possibility of their oscillations
}

\section{Oleg Kosmachev*}

VBLHEP JINR

E-mail: kosetheor.jinr.ru

Early we found three types of equations for unstable leptons. Two of them are connected with the unstable charged leptons $\left(\mu^{ \pm}, \tau^{ \pm}\right)$, the third is connected with a massive unstable neutrino. Analysis of tau lepton group (further group $\Delta_{3}$ ) points to existence of doubles to $\tau^{ \pm}$-leptons, that is $\left(\tau^{*}\right)^{ \pm}$-leptons. Together they form a quartet state, or a multiplet. Existence of $\tau$ and $\tau^{*}$ is connected with that the group $\Delta_{3}$ has two nonequivalent irreducible representations with dimension equal to four. Each of two nonequivalent representations describes a particle and antiparticle like Dirac's equation. Particles from different nonequivalent representations are not identical. Their quantum numbers coincide not completely. Therefore in this case it is possible to observe three various wave phenomena. Identical particles from the same nonequivalent representation can interfere. Particle and antiparticle from the same nonequivalent representations can annihilate. The leptons from different nonequivalent representations of the same quartet can oscillate.

XXII International Baldin Seminar on High Energy Physics Problems September 10-15, 2014

JINR, Dubna, Russia

\footnotetext{
*Speaker.
} 


\section{Introduction}

Lepton sector is recognized in modern particle physics as a key area and connecting link with astrophysics and cosmology. The increasing diversity of experimental facts beginning make high demands to description of the lepton sector as a whole. Advanced requirements concerning to coordination with fundamental principles, strict conclusion, internal consistency of sector, taking into account all it components and maximal freedom from phenomenological assumptions. Classical articles [1] and [2] serve as an examples in this case.

In resent years we have developed a holistic approach for the description of lepton sector [3], [4], [5]. The result is a complete and closed set of equations for massive and massless, charged and neutral, stable and unstable leptons in the framework of the Lorentz group and five initial assumptions. The completeness of the set of equations means it is impossible to complete it in any of classes of equations massive or massless, stable or unstable, charged or neutral. It is naturally to call our approach as the Dirac algorithm [6]. Its main feature looks like the answer to the question - what accurate and meaningful result can be obtained in the lepton sector, based on a few assumptions, non of which can be rejected? Those were found five. The criterion of impossibility to be rejected means here a minimum of assumptions, without which it is impossible to derive Dirac equation.

The initial suppositions are:

1. the equations must be invariant and covariant under homogeneous Lorentz transformations taken into account all four connected components;

2. the equations must be formulated on the base of irreducible representations of the groups determining every lepton equation;

3. conservation of four-vector of probability current must be fulfilled and fourth component of the current must be positively defined;

4. the lepton spin is supposed equal to $1 / 2$;

5. every lepton equation must be reduced to Klein-Fock-Gordon (KFG) equation;

It is easy to see that the set of assumptions is not excessive. It follows also from the algorithm that if a quantum object has the property to be a particle, it should be described by the equation of Dirac type. If the object has in addition wave properties, then its equation must be reduced to an equation of (KFG) type. This is equation of wave front propagation including de Broglie waves also. Dirac's decomposition [1] of (KFG) equation into two factors, every of which describes a particle, is concrete implementation of the wave-particle duality. It is easy to see that in addition to the Dirac algorithm here the Heisenberg idea is realized about the need to build a theory not on the basis of fundamental particles, but on the basis of fundamental symmetries.

\section{Notes on the stable lepton equations}

Articles [1], [2] and [7] created sufficient conditions for unified formulation of stable lepton equations. Each of the equation associated with it own group. All they are not isomorphic to each 
other. Therefore, the structure of each lepton equation is individual. Structure means here the presence of constituents that allow us to distinguish one equation from the other and to form on their basis operators that admit physical interpretation. Four connected components of the Lorentz group are the minimal substructures for all stable lepton equations. They are represented in the wave equations by means of infinitesimal form, they have the first weight number $l_{0}=1 / 2$ and they are Lee algebras for connected components. On the other hand this subgroups are maximal invariant subgroup also. We denote them as following:

1. group $d_{\gamma} \rightarrow$ is related to proper orthochronous representation;

2. group $f_{\gamma} \rightarrow$ ia related to improper orthochronous, $\langle P\rangle$ - conjugate representation;

3. group $b_{\gamma} \rightarrow$ is related to proper antichronous, $\langle T\rangle$ - conjugate representation;

4. group $c_{\gamma} \rightarrow$ is related to improper antichronous, $\langle P T\rangle$ - conjugate representation.

All subgroups are connected among themselves by discrete transformations $\langle T\rangle,\langle P\rangle,\langle P T\rangle$.

The listed subgroups have order equal to 16 and each is generated by three generators. The structure of stable lepton equations is very simple. All they have order equal to 32 . Therefore, the corresponding lepton do not disintegrate.

One of the condition for formulation stable lepton equations is the presence of four generators. Three of them must anticommute, thereby ensuring the presence of one or another connected components as a subgroup of 16 order. If the fourth generator to choose anticommutative with first three, we get equations for massive leptons. If the fourth generator commutes with first three, we get equations for massless leptons. The only requirement is that each generator of any equation boils dawn to the fact that it order should be two or four. Only in this case the result is value of spin equal to $1 / 2$. Simple calculations show [5] that changing only the order of generators (two or four) we can get a complete set of 32 order group, suitable for describing equations for stable leptons.

A single relativistic basis and individual differences of wave equations has lead to the structural formations - doublet, singlet and quartet lepton states. Doublets are states associated with equations that describe only particle and antiparticle. The singlets are states that do not have antiparticles. The quartets are the states that within a single group de scribe two doublet states, i.e. two pairs of particle-antiparticle. The structure here is the support of individual observable properties. It is expression of causality. Only due to the structure we receive opportunity to associate properties and quantum numbers of lepton with evident or hidden carriers of required characteristics.

The set of initial assumptions together with well known group theoretical limitations and requirements allows us to get five and only five equations similar to Dirac one, i.e. groups of equations for stable leptons. Their structural composition, i.e. the set of subgroups $d_{\gamma}, b_{\gamma}, c_{\gamma}, f_{\gamma}$ looks like this:

1. The Dirac equation $D_{\gamma}(I I):\left\{d_{\gamma}, b_{\gamma}, f_{\gamma}\right\}$

2. The equation for a doublet of massive neutrinos $D_{\gamma}(I):\left\{d_{\gamma}, c_{\gamma}, f_{\gamma}\right\}$ 
3. The equation for a quartet of massless neutrinos -

$D_{\gamma}(I I I):\left\{d_{\gamma}, b_{\gamma}, c_{\gamma}, f_{\gamma}\right\}$

4. The equation for a massless $T$-singlet -

$D_{\gamma}(I V):\left\{b_{\gamma}\right\}$

5. The equation for a massless $P$-singlet -

$D_{\gamma}(V):\left\{c_{\gamma}\right\}$.

Individuality of stable equation structures are obvious.

\section{Unstable leptons}

Unstable leptons by the fact of its existence and experimentally observable distinctions from stable one to prove the incompleteness of their theoretical description. The theory is forced under pressure of experiments to ascribe unstable leptons own quantum numbers, but it can not indicate that is the carriers of these numbers. Taking the distinctions of these two lepton types, the theory at the beginning proclaimed $(\mu-e)$-universality and then began speaking on $(\mu-e)$-problem [8]. Thus, the problem of unstable lepton description was recognized as an unresolved task.

Further extension of the lepton set for account of unstable one can be associated with involvement of fifth generator. It is possible to conserve the covariance of equations, if to choose fifth generator anticommutative with respect four previous. Reasons for this are as follows. The product of five anticommuting generators $\left(\Gamma_{6}=\Gamma_{1} \Gamma_{2} \Gamma_{3} \Gamma_{4} \Gamma_{5}\right)$ is the center of the group, i.e. it is an element multiple to unity. So we can use the fifth degree of freedom in stable equations and connect $\Gamma_{5}$ (by means of $\Gamma_{6}$ ) with mass number $m$. The covariance is conserved. The equations are obtained here more complex structures than stable one.

It was found that the initial assumption allow the formulation of three types of wave equations. Two of them are related with unstable charged leptons $(\mu, \tau)$, the third is related with a massive unstable neutrino. On the basis of structural differences naturally arose the distinctions between stable and unstable leptons.

Structural components for unstable leptons are the maximal subgroups which allow us easily and uniquely to distinguish between structure of one unstable group from another. They, on one hand, are different combinations of the same connected components and at the same time they are groups of stable leptons embedded into the unstable groups. So the extension of the Dirac group $\left(D_{\gamma}(I I)\right)$ by means of one anticommuting generator $\Gamma_{5}$ (such that $\Gamma_{5}^{2}=I$ ) leads to $\Delta_{1}$ group. This group has structural invariant [9], [5] equal to $\operatorname{In}\left[\Delta_{1}\right]=-1$. If one to extend Dirac group with the generator $\Gamma_{5}$ such that $\Gamma_{5}^{2}=-I$, than we obtain group $\Delta_{3}$. Structural invariant in this case equal to $\operatorname{In}\left[\Delta_{3}\right]=0$. Finally, the extension of massive neutrino group $D_{\gamma}(I)$ by means of generator $\Gamma_{5}^{2}=-I$ leads to the group $\Delta_{2}$ with the structural invariant equal to $\operatorname{In}\left[\Delta_{1}\right]=1$.

It is well known [1] the defining relations for the Dirac group looks as

$$
\gamma_{\mu} \gamma_{v}+\gamma_{v} \gamma_{\mu}=2 \delta_{\mu v}, \quad \mu, v=1,2,3,4
$$

Taking in to account above mentioned definitions we can write following expressions. 
Group $\Delta_{1}$ has the following defining relations:

$$
\Gamma_{\mu} \Gamma_{v}+\Gamma_{v} \Gamma_{\mu}=2 \delta_{\mu v}, \quad(\mu, v=1,2,3,4,5)
$$

As a result we obtain the following composition:

$$
\Delta_{1}\left\{D_{\gamma}(I I), \quad D_{\gamma}(I I I), \quad D_{\gamma}(I V)\right\}, \quad \operatorname{In}\left[\Delta_{1}\right]=-1 .
$$

Group $\Delta_{3}$ has the following defining relations:

$$
\begin{aligned}
& \Gamma_{s} \Gamma_{t}+\Gamma_{t} \Gamma_{s}=2 \delta_{s t}, \quad(s, t=1,2,3,4), \\
& \Gamma_{s} \Gamma_{5}+\Gamma_{5} \Gamma_{s}=0, \quad(s=1,2,3,4), \\
& \Gamma_{5}^{2}=-I .
\end{aligned}
$$

It follows from here:

$$
\Delta_{3}\left\{D_{\gamma}(I I), \quad D_{\gamma}(I), \quad D_{\gamma}(I I I)\right\}, \quad \operatorname{In}\left[\Delta_{3}\right]=0 .
$$

Group $\Delta_{2}$ has the following defining relations:

$$
\begin{aligned}
& \Gamma_{s} \Gamma_{t}+\Gamma_{t} \Gamma_{s}=2 \delta_{s t}, \quad(s, t=1,2,3), \\
& \Gamma_{s} \Gamma_{4}+\Gamma_{4} \Gamma_{s}=0, \quad(s=1,2,3), \\
& \Gamma_{4}^{2}=-I . \\
& \Gamma_{u} \Gamma_{5}+\Gamma_{5} \Gamma_{u}=0, \quad(u=1,2,3,4), \\
& \Gamma_{5}^{2}=-I .
\end{aligned}
$$

We obtain in this case:

$$
\Delta_{2}\left\{D_{\gamma}(I), \quad D_{\gamma}(I I I), \quad D_{\gamma}(V)\right\}, \quad \operatorname{In}\left[\Delta_{2}\right]=1 .
$$

All three groups have its own structures.

One can show that $\Delta_{1}$ and $\Delta_{3}$ are related to the equations for charged particles $\mu$ and $\tau$ respectively. Group $\Delta_{2}$ is related to equation for massive, unstable neutral particle. All three groups have some common properties. Their order is equal to 64 . The center of each group contains four elements and each has 34 conjugate classes. As a result, every of groups has 32 one-dimensional irreducible representations and two nonequivalent four-dimensional representations. In addition, each of groups is composed by three and only three maximal invariant subgroups. Their order is equal to 32. Each of these subgroups is isomorphic to one of five groups of stable leptons. The set of thirty second order subgroups in every $\Delta$-groups is its own nonrecurrent. The centers of the groups $\Delta_{1}$ and $\Delta_{2}$ have the same compositions $I,-I, I,-I$. The center of the $\Delta_{3}$-group has a different composition $I,-I, i I,-i I$. This distinction leads to the fact that nonequivalent four-dimensional representations are associated with a separate description of particle and antiparticle for group $\Delta_{1}$ and $\Delta_{2}$. In the $\Delta_{3}$-group each nonequivalent representation is associated with the doublet equation, i.e.they describe a particle and antiparticle like dirac equation. By definition the particles from different nonequivalent representations are not identical. 


\section{Peculiarities of the equations for description of tau-leptons}

Let us choose four Hermitian generators on the basis of defining relation (3.4) $\Gamma_{1}, \Gamma_{2}, \Gamma_{3}, \Gamma_{4}$. They are generators of the Dirac equation $D_{\gamma}(I I)$. This is always possible because taken separately group $D_{\gamma}(I I)$ and $\Delta_{3}$ have four dimension irreducible representations. The fifth generator $\Gamma_{5}$ is antihermitian by definition. With their help, the wave equation on the basis of $\Delta_{3}$ can be written in the form

$$
i \hbar \Gamma_{5} \partial \psi / \partial t=\left(-i \hbar c \Gamma_{1} \partial / \partial x-i \hbar c \Gamma_{2} \partial / \partial y-i \hbar c \Gamma_{3} \partial / \partial z+m c^{2} \Gamma_{4}\right) \psi
$$

Multiplying equation (4.1) from the left by $\Gamma_{5}^{-1}$, we obtain equality

$$
i \hbar \partial \psi / \partial t=\left(-i \hbar c \Gamma_{5}^{-1} \Gamma_{1} \partial / \partial x-i \hbar c \Gamma_{5}^{-1} \Gamma_{2} \partial / \partial y-i \hbar c \Gamma_{5}^{-1} \Gamma_{3} \partial / \partial z+m c^{2} \Gamma_{5}^{-1} \Gamma_{4}\right) \psi .
$$

It is obvious that four operators $\bar{\alpha}=\left\{\alpha_{1}=\Gamma_{5}^{-1} \Gamma_{1}, \alpha_{2}=\Gamma_{5}^{-1} \Gamma_{2}, \alpha_{3}=\Gamma_{5}^{-1} \Gamma_{3}\right\}$ and $\beta=\Gamma_{5}^{-1} \Gamma_{4}$ are Hermitian. Equation (4.2) by virtue of the form and properties of it terms satisfies conservation of probability current

$$
\partial\left(\psi^{\dagger} \psi\right) / \partial t=-c \cdot \operatorname{div}\left(\psi^{\dagger} \bar{\alpha} \psi\right)
$$

where $\psi^{\dagger}$ denotes hermitian conjugate solution and the value of $\bar{j}=c \cdot \operatorname{div}\left(\psi^{\dagger} \bar{\alpha} \psi\right)$ is a flow of probability. Thus a density of probability satisfies requirement of positive definiteness $\left(\psi_{1}^{*} \psi_{1}+\right.$ $\left.\psi_{2}^{*} \psi_{2}+\psi_{3}^{*} \psi_{3}+\psi_{4}^{*} \psi_{4}\right)$. Here $\psi_{1}^{*}$ denotes complex conjugation of value $\psi_{1}$.

Similarly, it turns the covariant formulation of the equation. We multiply (4.1) from the left by $\Gamma_{4}$ and transfer all additives to the left side

$$
\left(i \hbar \Gamma_{4} \Gamma_{5} \partial / \partial t+i \hbar c \Gamma_{4} \Gamma_{1} \partial / \partial x+i \hbar c \Gamma_{4} \Gamma_{2} \partial / \partial y+i \hbar c \Gamma_{4} \Gamma_{3} \partial / \partial z-m c^{2}\right) \psi=0 .
$$

One can verify by direct calculation that the four generators $\left(\Gamma_{4} \Gamma_{5}\right),\left(\Gamma_{4} \Gamma_{1}\right),\left(\Gamma_{4} \Gamma_{2}\right),\left(\Gamma_{4} \Gamma_{3}\right)$ generate the Dirac group $\left(D_{\gamma}(I I)\right)$. The first generator is hermitian, other are antihermitian. One can verify that the same subgroup $\left(D_{\gamma}(I I)\right)$ may be generated by another set of four generators, each of which is Hermitian: $\Gamma_{4}^{\prime}=\left(\Gamma_{4} \Gamma_{5}\right), \Gamma_{1}^{\prime}=\left(\Gamma_{1} \Gamma_{5}\right), \Gamma_{2}^{\prime}=\left(\Gamma_{2} \Gamma_{5}\right), \Gamma_{3}^{\prime}=\left(\Gamma_{3} \Gamma_{5}\right)$. Introducing the notation $x_{4}=i c t$ we obtain the usual expression for the Dirac type equation in covariant form

$$
\Gamma_{\mu}^{\prime} \partial \psi / \partial x_{\mu}+(m c / \hbar) \psi=0, \quad(\mu=1,2,3,4) .
$$

Equations (4.2) and (4.5) are evidence that each non-equivalent representation of the $\Delta_{3}$ group is associated with the description of particle and antiparticle like Dirac equation. If the generators of equations (4.4) to replace $\Gamma_{5} \rightarrow-\Gamma_{5}$, leaving $\Gamma_{1}, \Gamma_{2}, \Gamma_{3}, \Gamma_{4}$ without changes, then we get equation for another nonequivalent representation.

$$
\Gamma_{\mu}^{\prime} \partial \psi / \partial x_{\mu}-\left(m^{*} c / \hbar\right) \psi=0, \quad(\mu=1,2,3,4) .
$$

Every of equations(4.5) and (4.6) is reduced to Klein-Fok-Gordon equation separately. Therefore, we have no grounds to claim that $m=m^{*}$. However, in any case, we get two pairs of non-identical particles.

Reduction of equations for particles to the wave equation of (KFG) type is checking their wave properties. Particles of non-equivalent of a single quartet also exhibit wave properties in form more complex than the usual (KFG) equation. In this case, in addition to the normal derivatives of the 
second order $\left(\partial^{2} / \partial t^{2}, \partial^{2} / \partial x^{2}, \ldots\right)$ addition members appear. They can be of two types. If $m=m^{*}$, than we obtain elements with mixed derivatives apart from usual terms of (KFG) equation.

$$
2 \Gamma_{5} \Gamma_{1} \partial^{2} / \partial x \partial t+2 \Gamma_{5} \Gamma_{2} \partial^{2} / \partial y \partial t+2 \Gamma_{5} \Gamma_{3} \partial^{2} / \partial z \partial t
$$

If $m \neq m^{*}$, than we obtain in addition terms with derivatives of the first order of following type

$$
\left(m-m^{*}\right) \Gamma_{1} \Gamma_{4} \partial / \partial x+\left(m-m^{*}\right) \Gamma_{2} \Gamma_{4} \partial / \partial y+\left(m-m^{*}\right) \Gamma_{3} \Gamma_{4} \partial / \partial z
$$

These results are qualitative indications on possibility of oscillations. They are based on very general principles.

\section{Summary and outlook}

The existence of a quartet state of tau-leptons, if it gets experimental confirmation, will allow us to understand the different manifestations of wave nature of particles from single point of view. Identical particles can interfere. Particles and antiparticles annihilate if they are related to one doublet equation. Leptons belonging to different inequivalent representations, oscillate, if they are associated with a single quartet group.

The structure of the tau-lepton group together with the other two groups of unstable leptons indicates internal self-consistency of lepton sector as a whole, obtained on the basis of proposed algorithm. Indeed, the extension of two groups of stable leptons using fifth generator has lead three different groups. Moreover the structural composition of all three groups are different and contains particular set of group stable leptons as substructures and nothing beyond them. In this case, it is possible to speak not only about the completeness but also about closure of the resulting set of equations for all lepton sector.

The study of the structure of tau-leptons, free from excessive phenomenological assumption, has special meaning. Tau-leptons decay through many channels, including purely leptonic and channels involving hadrons in the final states. Such situation makes this research domain unique to study the structure of leptons and hadrons on a single relativistic basis. If to speak on a perspective of a detailed study of single physical vacuum or about the problem of formation of the complex on the basis of simple, it will be hardly successful without a unified description of both leptonic and hadronic sectors.

\section{References}

[1] Dirac P., Proc.Roy. Soc. A vol.117,610 (1928).

[2] Pauli W., Handbuch der Physik, (Verlag Julius Springer, Berlin) v.24, p. 226 (1933).

[3] Kosmachev O., Representations of the Lorentz group and classification of stable leptons: Preprint JINR, ï£;2-2006-6 (Dubna, 2006). (in russian)

[4] Gusev A, Kosmachev O., Phys. Part. Nucl. Lett. V.5, 67 (2008).

[5] Kosmachev O. Phys. Part. Nucl. Lett. V.7, 85 (2010). 
[6] Kosmachev O. "Dirac algorithm and elemetary particle physics". Proceedings of the Fifteenth Lomonosov conference on elementary particle physics. Moscow, Russia 18-24 August 2011. World Scientific.

[7] Majorana E., Il Nuovo Cimento v.14, 171 (1937).

[8] Komar A., Proceedings of seminar on $(\mu-e)$-problem. Moscow, "Nauka" 1974, PP 53-70.

[9] Lomont J., Applications of finite groups. Academic Press, New York, London: 1959, P.51. 